\title{
Hydration: Knowledge, Attitudes, and Practices of UK Dietitians
}

\author{
Pauline Douglas, ${ }^{1,2}$ Lauren Ball, ${ }^{2,3}$ Lynn McGuffin, ${ }^{1,2}$ Celia Laur, ${ }^{2}$ Jennifer Crowley, ${ }^{2,4}$ \\ Minha Rajput-Ray, ${ }^{2}$ Joan Gandy, ${ }^{2,5}$ and Sumantra Ray ${ }^{2,6}$ \\ ${ }^{1}$ Northern Ireland Centre for Food and Health, University of Ulster, Coleraine BT52 1SA, UK \\ ${ }^{2}$ UK Need for Nutrition Education/Innovation Programme in Partnership with the Medical Research Council Human Nutrition \\ Research Cambridge, and the British Dietetic Association, c/o Elsie Widdowson Laboratory, Cambridge CB1 9NL, UK \\ ${ }^{3}$ Centre for Health Practice Innovation, Griffith University, Southport, QLD 4222, Australia \\ ${ }^{4}$ School of Medical and Health Sciences, University of Auckland, Private Bag 92019, Auckland 1142, New Zealand \\ ${ }^{5}$ Dietetics Department, University of Hertfordshire, Hatfield Hertfordshire AL10 9AB, UK \\ ${ }^{6}$ Cambridge University Hospitals and School of Clinical Medicine, c/o Elsie Widdowson Laboratory, Cambridge CB1 9NL, UK
}

Correspondence should be addressed to Pauline Douglas; pl.douglas@ulster.ac.uk and Sumantra Ray; sumantra.ray@mrc-hnr.cam.ac.uk

Received 5 November 2014; Accepted 23 December 2014

Academic Editor: Friedrich Paulsen

Copyright (C) 2015 Pauline Douglas et al. This is an open access article distributed under the Creative Commons Attribution License, which permits unrestricted use, distribution, and reproduction in any medium, provided the original work is properly cited.

\begin{abstract}
Aim. The aim of this study was to investigate dietitians' knowledge, attitudes, and practices (KAP) regarding hydration and patient care. Methods. A cross-sectional online survey was administered to UK dietitians via the British Dietetic Association monthly newsletter and included 18 items on hydration knowledge $(n=8)$, attitudes $(n=4)$, and practices $(n=6)$. KAP scores were calculated by adding the total number of correct knowledge responses and by ranking attitude and practice responses on a Likert scale. Results. 97 dietitians completed the online survey and displayed varying levels of KAP regarding hydration and patient care. The mean unweighted scores were knowledge $5.0( \pm 1.3)$ out of 8 ; attitude $13.9( \pm 1.3)$ out of 16 ; practice $14.9( \pm 2.6)$ out of 24 . Dietitians appeared to be guided by clinical reasoning and priorities for nutrition care. Conclusions. There may be scope to further assess and potentially enhance the KAP of dietitians regarding hydration and patient care. Innovative approaches to hydration promotion are warranted and may include focusing on dietitians' personal hydration status, increasing communication with other healthcare professionals, and partnering with patients to take a proactive role in hydration monitoring.
\end{abstract}

\section{Introduction}

Hydration is a recognised determinant of health status for all population groups [1]. Mild dehydration can have negative health effects such as impaired physical function and cognitive decline [2]. There is limited understanding of the extent to which the hydration status of population groups increases health-related costs. There is, however, a growing evidence base of the use of health economics models for specific disease modalities $[3,4]$. Dehydration is recognized as a component of malnutrition, for which the United Kingdom (UK) health-related costs are estimated to be at least $£ 13 \mathrm{bn}$ annually [5]. As a result, national guidance widely encourages optimal hydration in UK hospital and community settings $[6,7]$.
Guidance exists for the promotion of hydration in UK hospitals [8]. The guidance provides practical advice for health care staff in England and Wales on how to minimise the risk and potential harm that dehydration can cause and offers solutions to improve the provision of fluids to patients in hospitals. Despite this guidance, it has been reported that patients continue to lack access to fresh drinking water and continued efforts to promote optimal hydration are needed [9]. Furthermore, over $90 \%$ of malnourished patients are cared for in community settings [10], suggesting that continued efforts for hydration promotion should occur in both hospital and community settings.

Healthcare professionals (HCPs) are expected to engage in best-practice care to address nutrition-related conditions in a multidisciplinary manner [11]. Registered dietitians are 
HCPs who assess, diagnose, and treat nutritional problems for individuals in the UK [12]. It is recommended that dietitians take a coordinated and integrated approach to addressing the nutritional care of patients, including promotion of optimal hydration, and lead on relevant knowledge transfer to other HCPs [13]. Dietitians should be competent in assessing patients' hydration requirements, developing strategies to meet hydration needs, and providing user outcome focussed services in all care settings as part of a holistic integrated package of care [14]. However, the competence of UK dietitians regarding hydration and patient care has not been studied.

The knowledge, attitudes, and practices (KAP) of HCPs have been widely investigated as indicators of behaviours [15-17]. Investigating the KAP of UK dietitians regarding hydration will inform strategies to support optimal hydration of patients in hospital and community settings. These strategies are important due to the recognised influence of hydration status on health outcomes [1] and will contribute to a reduction in health-related costs [18]. The aim of this study, therefore, was to investigate dietitians' KAP regarding hydration and patient care.

\section{Methods}

2.1. Overview. This study utilised a cross-sectional online survey to describe dietitians' KAP regarding hydration and patient care. The survey was conducted before the British Dietetic Association fluid factsheet was released in 2014. The study was undertaken as part of a larger project on hydration education in health care, and was exempt from ethical approval due to the nonsensitive, anonymous, educational nature of the survey.

2.2. Survey Instrument. A cross-sectional online survey (SurveyMonkeyPro) was developed following a review of relevant scientific literature (developed into evidence tables), published texts, "grey" literature, and expert opinion from dietitians, doctors, and hydration experts. Topics requiring investigation were categorised into key areas: facts regarding hydration, dehydration and fluid intake, kidney function and associated conditions, cognitive function, vulnerable groups regarding hydration (e.g., dysphagia and older adults), hydration assessment and advice in practice, personal and clinical attitudes towards hydration, and perceived importance of hydration training.

The survey was piloted on a range of HCPs at various levels including medical students, junior doctors, and GPs and reviewed by GPs, GP trainers, dietitians, scientists, and hydration experts for feedback on the interpretation and understanding of survey items. Recommendations to survey content, wording, and layout were completed prior to data collection. The finalised survey included 18 items on knowledge $(n=8)$, attitudes $(n=4)$, and practices $(n=$ 6 ), and each practice question also included an open ended textbox for respondents to justify their response. The survey was intended to take approximately five minutes to complete following recommendations by the GP reviewers that a short
TABLE 1: Dietitians' knowledge of hydration and patient care $(n=$ 97).

\begin{tabular}{lc}
\hline Knowledge area & $\begin{array}{c}\text { Proportion of participants } \\
\text { answering correctly } \\
n(\%)\end{array}$ \\
\hline Definition of dehydration & $74(76)$ \\
Physical signs of dehydration & $89(92)$ \\
Impact on performance tasks & $91(94)$ \\
Fluid requirements for adults & $39(40)$ \\
Fluid requirements for older adults & $59(61)$ \\
Water content of foods and drinks & $34(35)$ \\
Water content of the human body & $44(45)$ \\
Recommended water intake & $53(55)$ \\
\hline
\end{tabular}

survey would promote participant completion. The survey was only available in English.

2.3. Participant Sampling. The potential participant pool was dietitians working in the UK who were registered with the British Dietetic Association in February 2014. Information about the survey was included in the monthly newsletter for February 2014, including a brief description of the study, assurance of confidentiality, link to complete the survey, and contact details of the research team.

2.4. Data Analysis. Data analysis was conducted using the SPSS statistical software package version 22. Frequency distributions were calculated for each survey item, as well as mean and range for participants' years of experience. Knowledge scores were calculated for each participant by adding up the total number of correct answers for the knowledge questions. Attitudes and practice scores were calculated for each participant by ranking each response on a scale from 1 to 4 where 1 indicated low attitude or infrequent practice and 4 indicated high attitude or frequent practice. Data are presented as mean ( \pm standard deviation).

\section{Results}

A total of 126 dietitians accessed the survey during the data collection period, and 97 (77\%) completed the survey. Participants were from a combination of hospital and community settings and had been working as a dietitian for a mean of 6 years (range of $0-40$ years). More than one third $(n=39$; $40 \%$ ) of participants reported being in their current post for $\leq 2$ years, $28 \%(n=27)$ for $3-5$ years, $11 \%(n=11)$ for $6-9$ years, and $21 \%(n=20)$ for $10+$ years.

3.1. Knowledge. Table 1 outlines the hydration knowledge of participating dietitians. The mean number of questions correctly answered was $5.0( \pm 1.3)$ out of 8 . Most dietitians were able to recognise the physical signs of dehydration (92\% correct) and knew the impact that dehydration has on performance tasks (94\% correct). However, fewer dietitians knew the water content of the human body ( $45 \%$ correct), 
TABle 2: Dietitians' attitudes towards hydration in patient care $(n=97)$.

\begin{tabular}{|c|c|c|}
\hline Attitude area & Response & $\begin{array}{c}\text { Proportion of participants' } \\
\text { responses } \\
n(\%)\end{array}$ \\
\hline \multirow{4}{*}{ Person responsible for managing hydration intake of patients } & Dietitian & $0(0)$ \\
\hline & Doctor & $0(0)$ \\
\hline & Patient & $0(0)$ \\
\hline & All of the above & $97(100)$ \\
\hline \multirow{4}{*}{ Risk of excess water consumption on health } & No risk & $0(0)$ \\
\hline & Minimal risk & $25(26)$ \\
\hline & Moderate risk & $59(61)$ \\
\hline & Significant risk & $13(13)$ \\
\hline \multirow{4}{*}{ Importance of hydration for kidney stones } & Very important & $65(67)$ \\
\hline & Somewhat Important & $24(25)$ \\
\hline & Unimportant & $0(0)$ \\
\hline & Very unimportant & $8(8)$ \\
\hline \multirow{4}{*}{ Importance of hydration education for dietitians } & Very important & $61(63)$ \\
\hline & Somewhat Important & $32(33)$ \\
\hline & Unimportant & $3(3)$ \\
\hline & Very unimportant & $1(1)$ \\
\hline
\end{tabular}

fluid requirements for adults $(40 \%$ correct), and the water content of foods and drinks (35\% correct).

3.2. Attitudes. Table 2 outlines the hydration attitudes of participating dietitians. The mean attitudes score was $14.0( \pm 1.3)$ out of a maximum score of 16 . All dietitians (100\%) reported that hydration management is the combined responsibility of HCPs and patients and recognised some degree of risk in consuming excess water. Nearly all participants (96\%) reported that hydration education for dietitians is important.

3.3. Self-Reported Practices. Table 3 outlines the hydration practices of participating dietitians. The mean practice score was $14.9( \pm 2.6)$ out of a maximum score of 24. Most dietitians (91\%) promoted hydration in standard care by encouraging intake of water and other beverages and reported wide variation in time spent promoting hydration to patients. Variations in practices were also apparent for promoting hydration to stroke patients and assessing urine colour. The majority of dietitians (58\%) rated their personal hydration practices as bad or average but reported using water dispensing facilities at their place of work.

Dietitians reported that it is important to promote liberal intakes of all fluids to facilitate compliance and maximise the likelihood of patients reaching optimal hydration status. The amount of time dietitians spent on hydration promotion was dependent on the nutritional priorities of patients. Free text responses from dietitians who never promoted hydration to stroke patients reported that it was either not applicable to their current post $(n=20)$ or that cognitive impairments of patients hindered communication $(n=1 ; n=2$ did not comment). Self-reported urine colour was deemed to be a practical and valid indicator of hydration status. The main barrier to dietitians consuming adequate fluids at work was lack of time and not remembering to drink.

\section{Discussion}

This study investigated the KAP of dietitians in the UK regarding hydration and patient care. This is important as dietitians are ideally placed to advise and educate on the benefits of appropriate hydration alongside nutrition [14, 19]. The dietitians in the current study displayed approaches to hydration and patient care that are realistic to practice settings. However, there were noted opportunities for improved hydration KAP.

The hydration knowledge of dietitians in this study appeared to be lacking. Whilst dietitians displayed very good understanding of physical signs of dehydration and its impact on cognitive performance, fewer dietitians provided correct answers to other knowledge questions, such as recommended water intakes and water content of food, drinks, and the human body. It is important to note that the evidence relating to specific hydration issues such as water content of foods and hydration requirements of patients is evolving [20, 21]. Based on the EFSA fluid intake from beverages recommendations, the most recent hydration guidelines promoted 8-10 glasses (200 mL glass) per day [22] which is higher than the previous guidelines of 6-8 glasses per day [23]. As a result, dietitians may have variable understanding of specific hydration issues and require further education to maintain clinical relevancy.

Participants appeared to recognise the need for improved hydration knowledge given that only $4 \%$ of participants regarded hydration education as unimportant. As a registered dietitian in the UK, it is a requirement to remain competent to practice $[14,24,25]$, thus requiring a need for career-long learning to maintain evidence-based knowledge and skills 
TABLE 3: Dietitians' self-reported practices regarding hydration in patient care $(n=97)$.

\begin{tabular}{|c|c|c|}
\hline Practice area & Response & $\begin{array}{c}\text { Proportion of participants' } \\
\text { responses } \\
n(\%)\end{array}$ \\
\hline \multirow{4}{*}{ Usual method of promoting hydration in standard care } & Not part of care & $7(7)$ \\
\hline & Encourage reduced caffeine intake & $1(1)$ \\
\hline & Encourage water intake only & $1(1)$ \\
\hline & Encourage water and other beverages & $88(91)$ \\
\hline \multirow{4}{*}{$\begin{array}{l}\text { Average time spent providing hydration advice in a } 4 \text {-hour } \\
\text { clinical session }\end{array}$} & 0 minutes & $4(4)$ \\
\hline & Between 0 and 10 minutes & $30(31)$ \\
\hline & Greater than 10 minutes & $32(33)$ \\
\hline & Unable to quantify & $31(32)$ \\
\hline \multirow{4}{*}{ Frequency of promoting hydration to stroke patients } & Never & $23(24)$ \\
\hline & Occasionally & $17(18)$ \\
\hline & Regularly & $22(22)$ \\
\hline & Always & $35(36)$ \\
\hline \multirow{4}{*}{ Frequency of assessing patients' self-reported urine colour } & Never & $6(6)$ \\
\hline & Occasionally & $36(37)$ \\
\hline & Regularly & $38(39)$ \\
\hline & Always & $17(18)$ \\
\hline \multirow{4}{*}{ Personal rating of hydration status at work } & Bad & $14(14)$ \\
\hline & Average & $43(44)$ \\
\hline & Good & $26(27)$ \\
\hline & Excellent & $14(14)$ \\
\hline \multirow{4}{*}{ Workplace access and use of water dispensing facilities } & Yes, and I use it & $64(66)$ \\
\hline & Yes, but I do not use it & $10(10)$ \\
\hline & No, but I would use if available & $22(23)$ \\
\hline & No, I would not use it & $1(1)$ \\
\hline
\end{tabular}

$[26,27]$. While the dietitians in this study recognized that nutrition and hydration are integral to optimise patient outcomes, the dietetics curriculum framework does not specifically mention hydration [14]. It has been said that water is the forgotten nutrient [8] and it could be suggested that it is important to be explicit in documentation to state nutrition and hydration together.

Dietitians in this study displayed positive attitudes towards hydration and its impact on prevention and patient care. They recognised the importance of a multidisciplinary approach to hydration promotion, particularly for at-risk patient groups and for dietitians to be well educated on hydration. However, it has been previously established that UK HCPs place less importance on hydration compared with counterparts in Mediterranean countries, which may be due to variations in climate [19]. Strategies to enhance a multidisciplinary approach to hydration care may lower the gap between UK HCPs and other countries by, for example, increased focus on the NHS nutrition and hydration awareness weeks [6].

Qualitative responses from free text boxes indicated that self-reported hydration practice was influenced by higher nutrition priorities for their patients. Therefore, despite the participants having a good attitude towards hydration care, this may not always translate into patient care. Less than half of the dietitians in this study reported good personal hydration while at work, with $24 \%$ indicating that this was due to lack of access to water dispensing facilities in their workplace. This is particularly pertinent for health care professionals working in a community setting and carrying out domiciliary visits where there will be a lack of access to water dispensing and bathroom facilities. The impact of dietitians' personal habits on their counselling practices has not been investigated; however, doctors with healthy personal habits or a desire to improve their own health are more likely to counsel patients [28-30]. Similarly, interventions that focus on medical students' personal nutrition behaviours have been shown to improve the frequency of nutrition counselling [31]. These studies suggest that emphasis should be given to dietitians' workplace hydration practices to facilitate improvements in their own hydration status and the hydration-related care provided to patients.

As previously mentioned, in some hospitals and practice settings there were no water dispensing facilities available for staff. This has been related to guidance on preventing legionella and pseudomonas outbreaks in care settings, leading to infection control guidance, strict use and service maintenance records, or withdrawal of dispensers [32, 33]. HCPs should be cognisant of sourcing water throughout the day which could be supported if the substantive evidence of 
how to maintain water dispensing facilities is appropriately applied [32-34].

Innovative approaches to promoting adequate fluid intake are required in UK hospitals and community settings [7]. One approach that has experienced positive outcomes is enhancing patients' participation in their health and medical care [35-37]. These studies report that patients are receptive to taking a proactive role in health care, which suggests that there are similar opportunities for hydration promotion initiatives utilising patient-participation. For example, initiatives could facilitate patients to self-monitor fluid balance, in order to reach targets set in collaboration with dietitians. A similar approach has been successfully trialled in Australia to enhance patients' protein and energy intake [38] and warrants further consideration.

Key limitations of the present study should be noted. Firstly, the strategy used to recruit participants may have resulted in some selection bias [39]. It is likely that those agreeing to participate may have had a particular interest in hydration and therefore may have resulted in an overestimation of dietitians' KAP. Secondly, a response rate could not be calculated as it is unknown how many dietitians read the newsletter/advertisements and chose not to participate. Similarly, questions relating to demographic characteristics were not included in the survey, which limited investigations into the representativeness of the participating dietitians in relation to the overall UK dietetic workforce. Finally, the self-reported nature of the survey may have resulted in participants providing more clinically desirable responses than a true reflection of their attitudes and practices.

There may be scope to further assess and potentially enhance the KAP of dietitians regarding hydration and patient care. The dietitians in the current study appear to be guided by clinical reasoning and priorities for nutrition care. There is also a potential opportunity to follow up this sample of dietitians to investigate whether release of the British Dietetic Association's fluid factsheet has influenced KAP regarding hydration. The current study suggests that promotion of optimal hydration for patients requires a broader focus involving patients and other HCPs. The NNEdPro Group is currently examining the KAP in relation to the hydration education of medical doctors and will continue this work with other HCPs to determine their need for further training. Innovative approaches to hydration promotion are warranted and may include focusing on dietitians' personal hydration status and their leadership role in educating other HCPs and partnering with patients to take a proactive role in hydration monitoring.

\section{Conflict of Interests}

Joan Gandy works as a consultant for Danone Waters.

\section{Authors' Contribution}

Pauline Douglas, Lynn McGuffin, Celia Laur, Minha RajputRay, Joan Gandy, and Sumantra Ray contributed to the conception and design of the project. Pauline Douglas, Celia
Laur, Lynn McGuffin, and Sumantra Ray designed the survey instrument. Lauren Ball and Jennifer Crowley conducted the data analysis and drafted the paper. All authors participated in finalisation of the paper.

\section{Acknowledgments}

The authors would like to thank Drs. Laurent Le Bellego, David Roos, and Liliana Jimenez for their contribution to the project and Drs. Stephen Gillam, Kate Earl, and Laura Fitzpatrick for their assistance. Special thanks are due to the British Dietetic Association for distributing the survey, as well as other NNEdPro key partners including the Cambridge University Hospitals/School of Clinical Medicine, the University of Ulster, and the UK Medical Research Council Human Nutrition Research unit in Cambridge, UK. The authors also thank all of those who assisted with piloting of this survey, the dietitians who completed the survey and those who provided support and feedback throughout the project. This project was supported by an education project by Danone Waters, of which Pauline Douglas and Sumantra Ray were coprincipal investigators and Lynn McGuffin, Celia Laur, Minha RajputRay, and Joan Gandy were named investigators. Sumantra Ray is also funded by the Medical Research Council.

\section{References}

[1] B. M. Popkin, K. E. D'Anci, and I. H. Rosenberg, "Water, hydration, and health," Nutrition Reviews, vol. 68, no. 8, pp. 439458, 2010.

[2] H. Gibson-Moore, "Hydration and health," Nutrition Bulletin, vol. 39, no. 1, pp. 4-8, 2014.

[3] Y. Lotan, I. B. Jiménez, I. Lenoir-Wijnkoop et al., "Primary prevention of nephrolithiasis is costeffective for a national healthcare system," BJU International, vol. 110, no. 11, pp. E1060E1067, 2012.

[4] Y. Lotan, I. Buendia Jiménez, I. Lenoir-Wijnkoop et al., "Increased water intake as a prevention strategy for recurrent urolithiasis: major impact of compliance on cost-effectiveness," Journal of Urology, vol. 189, no. 3, pp. 935-939, 2013.

[5] M. Elia and C. Russell, Combating Malnutrition: Recommendations for Action, BAPEN, Redditch, UK, 2009.

[6] British Association for Enteral and Parenteral Nutrition (BAPEN), Nutrition and hydration week, 2014, http://www .bapen.org.uk/news-and-media/news/nutrition-and-hydrationweek-2014.

[7] British Dietetic Association, The Nutrition and Hydration Digest: Improving Outcomes Through Food and Beverage Services, British Dietetic Association, Birmingham, UK, 2012.

[8] Royal College Nursing and National Patient Safety Agency, Water for Health: Hydration Best Practice Toolkit for Hospitals and Healthcare, Royal College Nursing, London, UK, 2009.

[9] Parliamentary and Health Service Ombudsman, "Care and compassion?" Report of the Health Service Ombudsman on 10 Investigations into NHS Care of Older People, Parliamentary and Health Service Ombudsman, London, UK, 2011.

[10] R. M. Leach, A. Brotherton, M. Stroud, and R. Thompson, "Nutrition and fluid balance must be taken seriously," British Medical Journal, vol. 346, article f801, 2013. 
[11] P. M. Kris-Etherton, S. R. Akabas, C. W. Bales et al., “The need to advance nutrition education in the training of health care professionals and recommended research to evaluate implementation and effectiveness," The American Journal of Clinical Nutrition, vol. 99, no. 5, pp. 1153S-1166S, 2014.

[12] British Dietetic Association, Dietitian, Nutritionist, Nutritional Therapist or Diet Expert? A Comprehensive Guide to Roles and Functions, British Dietetic Association, Birmingham, UK, 2014.

[13] British Dietetic Association, The Care of Nutritionally Vulnerable Adults in Community and All Health and Care Settings, British Dietetic Association, Birmingham, UK, 2012.

[14] British Dietetic Association, "A curriculum framework for the pre-registration education and training of dietitians," Birmingham, UK, 2013.

[15] K. L. Delucchi, B. Tajima, and J. Guydish, "Development of the smoking knowledge, attitudes, and practices (S-KAP) instrument," Journal of Drug Issues, vol. 39, no. 2, pp. 347-364, 2009.

[16] L. S. Eller, E. Kleber, and S. L. Wang, "Research knowledge, attitudes and practices of health professionals," Nursing Outlook, vol. 51, no. 4, pp. 165-170, 2003.

[17] T. Saleem, U. Khalid, S. Ishaque, and A. Zafar, "Knowledge, attitudes and practices of medical students regarding needle stick injuries," Journal of the Pakistan Medical Association, vol. 60 , no. 2, pp. 151-156, 2010.

[18] National Institute for Health and Clinical Excellence, Nutrition Support for Adults. Clinical Guideline 32, London, UK, 2006.

[19] J. E. Holdsworth, "The importance of human hydration: perceptions among healthcare professionals across Europe," Nutrition Bulletin, vol. 37, no. 1, pp. 16-24, 2012.

[20] European Food Safety Authority (EFSA), "Scientific opinion on dietary reference values for water," EFSA Journal, vol. 8, no. 3, p. 1459, 2010.

[21] R. L. Sharp, "Role of whole foods in promoting hydration after exercise in humans," Journal of the American College of Nutrition, vol. 26, no. 5, supplement, 2007.

[22] British Nutrition Foundation, Healthy Hydration Guide, 2010, http://www.nutrition.org.uk/healthyliving/hydration/healthyhydration-guide.

[23] Food Standards Agency, Drinking Enough?, 2010, http://tna .europarchive.org/20100929190231/http://www.eatwell.gov.uk/ healthydiet/nutritionessentials/drinks/drinkingenough/.

[24] British Dietetic Association, Code of Professional Conduct, British Dietetic Association, Birmingham, UK, 2008.

[25] Health and Care Professions Council, Standards of Proficiency, Dietitians, London, UK, 2013.

[26] Health and Care Professions Council, Continuing Professional Development and Your Registration, Health and Care Professions Council, London, UK, 2012, (updated 2014).

[27] Thematic Network for Dietetics, "DIETS: dietitians ensuring education teaching and professional quality (2010-2013)," in Guide to Lifelong Learning for Dietitians in Europe. How to Develop Your Professional Competence, A. D. Looy, Ed., Thematic Network for Dietetics, Plymouth, UK, 2013.

[28] E. Frank, J. Breyan, and L. Elon, "Physician disclosure of healthy personal behaviors improves credibility and ability to motivate," Archives of Family Medicine, vol. 9, no. 3, pp. 287-290, 2000.

[29] O. Hung, N. L. Keenan, and J. Fang, "Healthier personal habits of primary care physicians increase the likelihood of their recommending lifestyle modifications for their hypertensive patients that are consistent with the national guidelines," Circulation, vol. 125, abstract P136, 2012.
[30] K. B. Wells, C. E. Lewis, B. Leake, and J. E. Ware Jr., "Do physicians preach what they practice? A study of physicians' health habits and counseling practices," The Journal of the American Medical Association, vol. 252, no. 20, pp. 2846-2848, 1984.

[31] E. Frank, J. S. Carrera, L. Elon, and V. S. Hertzberg, "Predictors of US medical students' prevention counseling practices," Preventive Medicine, vol. 44, no. 1, pp. 76-81, 2007.

[32] Department of Health, "Water Systems: Health Technical Memorandum 04-01, Addendum: Pseudomonas aeruginosa-advice for augmented care units," Quarry Hill, UK, 2013.

[33] World Health Organization, Water Safety in Buildings, World Health Organization, Geneva, Switzerland, 2011.

[34] Public Health England, Draft Draft Guidelines for Collection and Interpretation of Results from Microbiological Examination of Food, Water and Environmental Samples from the Hospital Environment, Public Health England, London, UK, 2010.

[35] M. J. Crawford, D. Rutter, C. Manley et al., "Systematic review of involving patients in the planning and development of health care," British Medical Journal, vol. 325, no. 7375, pp. 1263-1265, 2002.

[36] S. Latimer, W. Chaboyer, and B. Gillespie, "Patient participation in pressure injury prevention: giving patient's a voice," Scandinavian Journal of Caring Sciences, vol. 28, no. 4, pp. 648-656, 2014.

[37] C. Mockford, S. Staniszewska, F. Griffiths, and S. HerronMarx, "The impact of patient and public involvement on UK NHS health care: a systematic review," International Journal for Quality in Health Care, vol. 24, no. 1, pp. 28-38, 2012.

[38] S. Roberts, B. Desbrow, and W. Chaboyer, "Feasibility of a patient-centred nutrition intervention to improve oral intakes of patients at risk of pressure ulcer: a pilot randomised control trial," Scandinavian Journal of Caring Sciences, Under Review.

[39] L. Neuman, Social Research Methods: Qualitative and Quantitative Approaches, Pearson International Edition, 6th edition, 2006. 

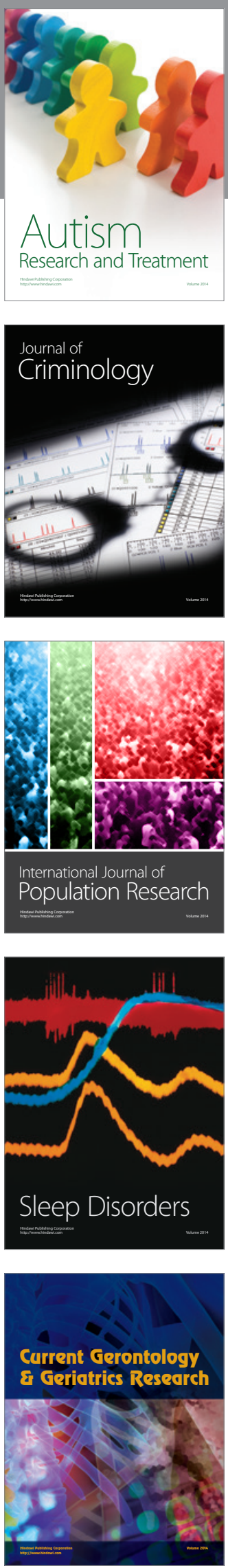
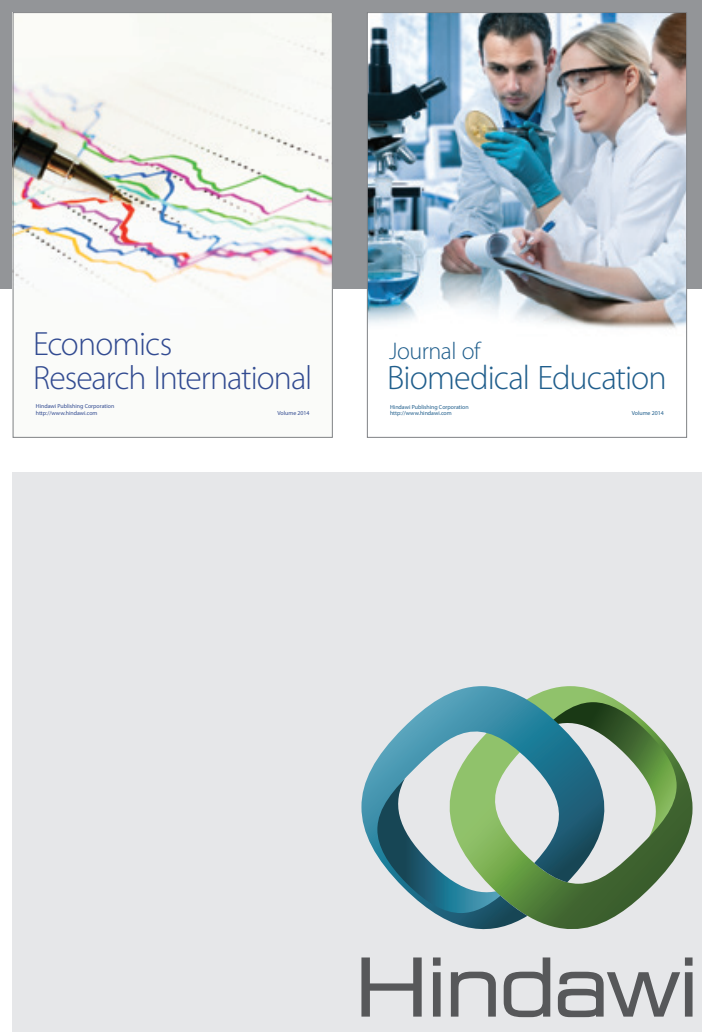

Submit your manuscripts at

http://www.hindawi.com
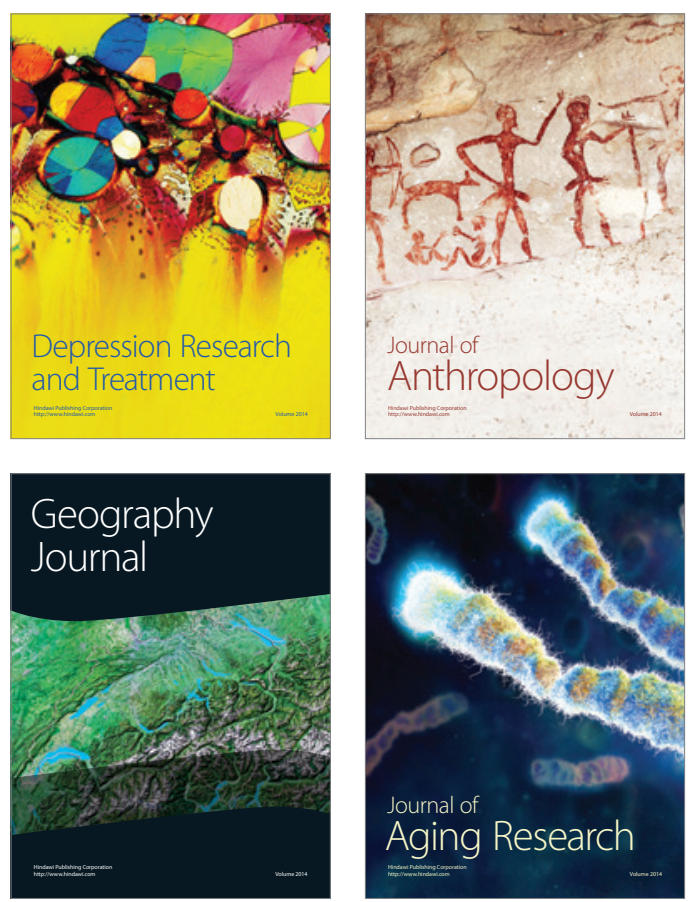
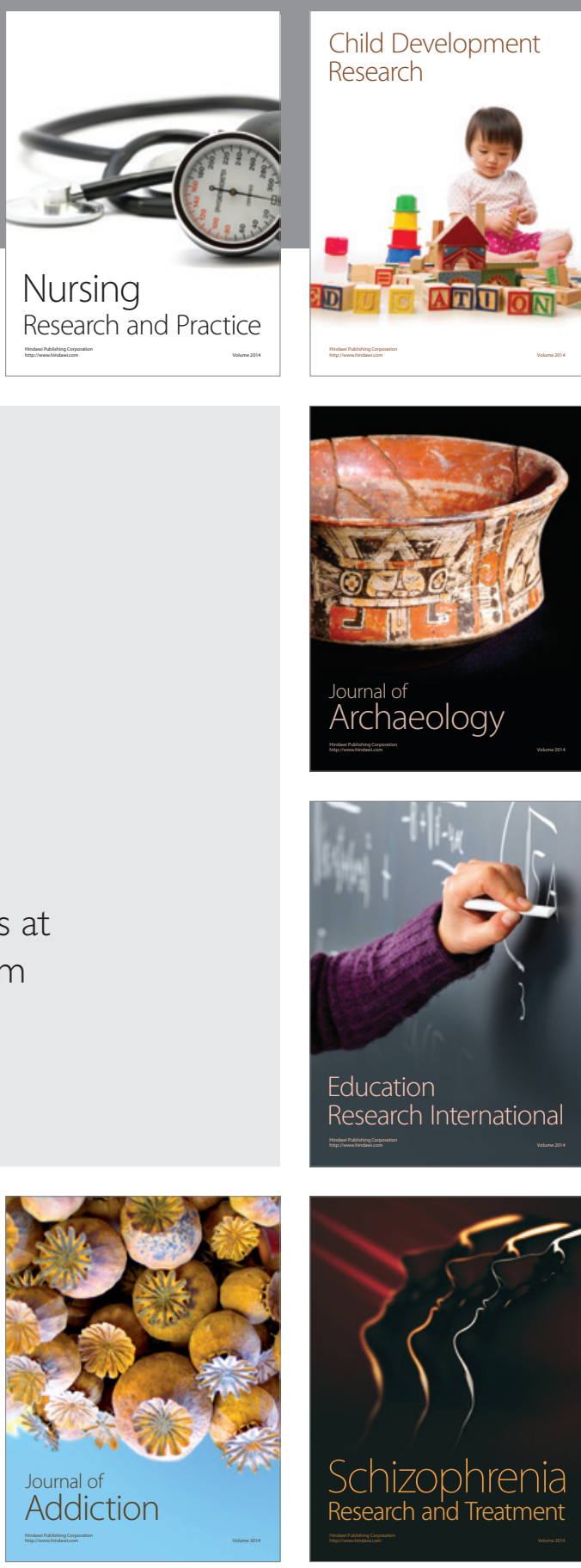

(D)
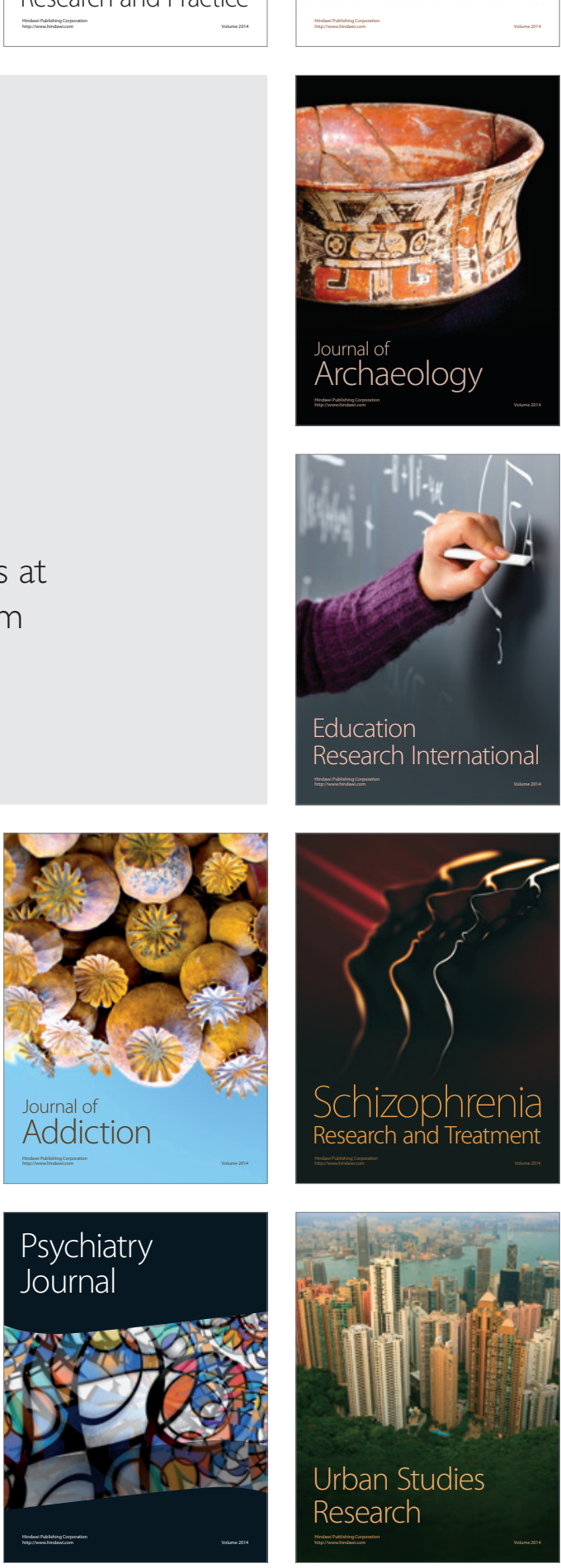